\title{
Solución de onda unidimensional por diferencias finitas
}

\author{
Francisco Barralaga $^{1}$ y Alejandro GalO ${ }^{2}$ \\ ${ }^{1}$ Escuela de Fúsica - UNAH, mail: francisco.barralaga@unah.edu.hn \\ ${ }^{2}$ Escuela de Física - UNAH, mail: alejandrogaloroldan@gmail.com
}

Recibido: 28 de febrero de 2017 / Aceptado: 30 de abril de 2017

\begin{abstract}
Resumen
The unidimensional wave equation is an imperative topic to be considered in the study of oscillatory phenomena, and mechanical vibrations. The analytical solution of this equation is addressed since the differential equations and partial derivatives field, as a topic, not always included in the engineer or science study program. Even more unprovable is that students from these careers, have the chance to study the numerical methods to solve this important equation.

In this context and with the objective of offer more auxiliary didactic options to approach this important topic, was developed a Java environment system which uses the computer simulation to visualize the convergence process of a numerical algorithm, defined in terms of the Finite Difference numerical method, to obtain the solution to the unidimensional wave equation. The user interface of the simulator system, FDTDM-EcOnda, allows the graphical comparability of the analytical and numerical solutions.

With this simulator is possible produce numerical value variation of the parameters and visualize the corresponding effect in the numerical solution involved. The simulator includes options to get curve adjustment and statically analysis to estimate the correlation between both solutions.
\end{abstract}

Keywords: Electromagnetism, Wave Equation, Finite Differences, Simulation

La ecuación de onda unidimensional es un tema obligado en el estudio de los fenómenos ondulatorios y de las vibraciones mecánicas. Su solución analítica se aborda en el campo de las ecuaciones diferenciales con derivadas parciales, un tema no siempre incluido en el pénsum de los estudiantes de ingenierías y ciencias. Aun menos posible es que los estudiantes de estas carreras tengan la oportunidad de estudiar la aplicación de métodos numéricos para obtener la solución de esta importante ecuación.

En este contexto y con el objetivo de proveer opciones didácticas auxiliares para el estudio de esta importante temática, fue desarrollado un sistema en un entorno basado en Java que hace uso de la simulación en computadora para visualizar el proceso de convergencia de un algoritmo numérico definido en términos del método de diferencias finitas, para dar solución a la ecuación de onda unidimensional. La interfaz gráfica de usuario del sistema simulador, FDTDM-EcOnda, permite comparar gráficamente la solución obtenida por el algoritmo numérico con la solución analítica.

Con el simulador es posible variar los parámetros del algoritmo numérico y verificar de inmediato los efectos de estos cambios en la solución numérica generada. El simulador incluye un módulo con opciones de ajuste de curvas y análisis estadístico con el que es posible estimar el grado de correlación entre ambas soluciones.

Palabras clave: Electromagnetismo, Ecuación de Onda, Diferencias Finitas, Simulación

\section{INTRODUCCIÓN}

$\Lambda$ MEDIDA que los lenguajes de programación lo permitieron, las aplicaciones destinadas a reforzar los procesos de enseñanza-aprendizaje, más allá del simple entretenimiento, han tenido siempre un lugar en el mercado de la educación. Cuando en los años 80 , se concluyó que la modalidad de enseñanza tutorial uno-a-uno resultaba tener ventajas sobre las opciones convencionales de enseñanza a grupos numerosos $[3$, los objetivos de las aplicaciones diseñadas para una enseñanza apoyada en computadora fueron redefinidos hacia la estructuración de una enseñanza con componentes de virtualidad.
Los cursos de física y matemáticas representan tradicionalmente una carga de estudio considerable para el estudiante. La Física, y en particular la Teoría Electromagnética en la que se abordan tópicos de solución de la ecuación de onda[11] 2], implican el desarrollo de habilidades que involucran la abstracción espacial y conceptual, y el manejo de un nivel matemático adecuado, necesarios para interpretar los modelos, conceptos y leyes consideradas, generados a partir de ecuaciones matemáticas, que representan campos vectoriales variantes con el tiempo, definidos en dos y tres dimensiones. La búsqueda de soluciones para mejorar la enseñanza de estos tópicos y la creación de auxiliares didácticos en su enseñanza, resultan de absoluta importancia. 
En la 44ava Conferencia de Decisión y Control del IEEE de 2005, y en la Conferencia Europea de Control del mismo año, fue presentada en Sevilla, España, una herramienta basada en Java que podía ser usada como interfaz de Simulink [5], una costosa herramienta de Matlab muy utilizada en procesos de simulación y control. Esta herramienta, Easy Java Simulations [7] (EJS por sus siglas en inglés), la cual es totalmente gratuita y que puede fácilmente ser descargada de su sitio web oficial [6]; ha sido utilizada para el modelamiento visual y numérico de fenómenos físicos, generando en cada caso simulaciones en computadora de muy buena calidad, para cuya implementación se requiere poco tiempo de programación; EJS representa una solución auxiliar para ambientes académicos con recursos limitados.

Se describe en este artículo a FDTDM-EcOnda, un sistema desarrollado en el ambiente de EJS que simula en computadora el proceso de solución numérica de una ecuación de onda unidimensional a partir de un algoritmo que aplica el Método de Diferencias Finitas con Dominio del Tiempo (FDTDM). El sistema simulador tiene opciones que permiten comparar gráficamente la solución obtenida por el algoritmo numérico con la solución analítica. El simulador además permite al usuario cambiar los parámetros del algoritmo numérico y visualizar los efectos de estos cambios en la solución generada. Se incluye en el simulador acceso a opciones de ajuste de curvas y análisis estadístico con lo que se estima el grado de correlación entre ambas soluciones.

\section{DESARROLLO DEL TEMA}

Considerando un sistema de coordenadas rectangulares, y denominando $\psi$ a la solución buscada, debemos resolver la ecuación siguiente

$$
\nabla^{2} \psi=\left(\epsilon_{0} \mu_{0}\right) \frac{\partial^{2} \psi}{\partial t^{2}}
$$

en la que $\psi$ es una función de la posición $r$ y del tiempo $t$, es decir $\psi(r, t)$, una ecuación tridimensional que representa a cualquiera de las seis componentes rectangulares de $E$ y $B$, siendo esta última la única ecuación escalar que necesita ser resuelta.

Nos concentraremos en la solución de esta ecuación a lo largo de uno de los tres ejes coordenados; digamos el eje $x$, es decir $\psi(x, t)$. En primer lugar, suponemos una solución particular para $\psi(x, t)$ con las siguientes características:

1. $\psi(x, t)$ es independiente de $z$ e $y$,

2. La solución será de la forma $\psi(r, t)=f(x-v t)+$ $g(x+v t)$

Si $\omega=x-v t$, entonces $f$, el primer término de $\psi(r, t)$, es función de $x$. En consecuencia, por la regla de la cadena se cumple que

$$
\frac{\partial \psi}{\partial x}=\frac{d \psi}{d \omega} \frac{\partial \omega}{\partial x}=\frac{d \psi}{d \omega}
$$

y por tanto,

$$
\begin{gathered}
\frac{\partial^{2} \psi}{\partial x^{2}}=\frac{d^{2} \psi}{d \omega^{2}} \frac{\partial \omega}{\partial x}=\frac{d^{2} \psi}{d \omega^{2}} \\
\frac{\partial \psi}{\partial t}=\frac{d \psi}{d \omega} \frac{\partial \omega}{\partial t}=-v \frac{d \psi}{d \omega} \\
\frac{\partial^{2} \psi}{\partial z^{2}}=-v \frac{d^{2} \psi}{\mathrm{d} \omega^{2}} \frac{\partial \omega}{\partial z}=v^{2} \frac{d^{2} \psi}{\mathrm{d} \omega^{2}}
\end{gathered}
$$

Al sustituir en la ecuación (5), se llega a una ecuación diferencial de derivadas parciales a la que se denomina ecuación de onda, cuya solución nos dará la expresión para $f$.

$$
\frac{\partial^{2} \psi}{\partial z^{2}}=\frac{1}{v^{2}} \frac{\partial^{2} \psi}{\partial t^{2}}
$$

Similarmente $g(x+v t)$ es una solución de la misma ecuación de onda, lo que muestra que la solución total de la misma está constituida por una onda que se propaga con rapidez $v$ hacia la derecha de $x, f(x-v t)$, juntamente con otra que lo hace hacia la izquierda, es decir, la porción $g(x+v t)$.

Si se trata de una onda electromagnética en el vacío, deberá cumplirse que,

$$
v=\frac{1}{\sqrt{\epsilon_{0} \mu_{0}}}
$$

Consideraremos la solución numérica de la ecuación de onda, ecuación (6). La ecuación (8) es esta misma ecuación planteada en una dimensión; en este caso, en el eje $\mathrm{x}$,

$$
\frac{\partial^{2} U(x, t)}{\partial t^{2}}-\alpha^{2} \frac{\partial^{2} U}{\mathrm{dx}^{2}}=0
$$

Si consideramos la evolución de esta ecuación a largo de un tramo L sobre el eje $\mathrm{x}$, entonces,

- $0 \leq x \leq L$,

- $t \geq 0$,

- $\alpha$ una constante que depende de las condiciones físicas particulares.

En este caso se consideran las siguientes condiciones iniciales y de frontera,

- $U(0, t)=U(L, t)=0$,

- $t \geq 0$

- $U(x, 0)=f(x)$, correspondiendo al pulso o perturbación inicial,

- $\delta U(x, 0)) / \delta t=g(x)$ 
- y con $0 \leq x \leq L$

Se aplica el método numérico más conocido para la solución de este tipo de ecuaciones, el método de Diferencias Finitas. La aplicación de este método requiere implementar el algoritmo que se describe posteriormente.

\section{A. Método de Diferencias Finitas con Dominio del Tiempo (FDTD)}

El método de Diferencias Finitas tiene como objetivo la aproximación de los términos de las ecuaciones diferenciales por medio de diferencias a las que se llega usualmente a través de desarrollos en series de Taylor. Las ecuaciones obtenidas se resuelven simultáneamente llegando así a una solución aproximada.

Si la ecuación no tiene dependencia del tiempo entonces el método se llama simplemente de Diferencias Finitas, (FDM por sus siglas en inglés), pero, si la situación implica considerar la dependencia con el tiempo, entonces el método es nombrado de Diferencias Finitas con Dominio Temporal (FDTD por sus siglas en inglés).

Se comienza por discretizar el espacio o vecindad en el que será resuelta la ecuación diferencial. Esto se hace usualmente mediante una cuadrícula que por simplicidad escogeremos cuadrada.

El desarrollo de una función cualquiera, $F(x)$ en series de Taylor alrededor de $x$ con una vecindad constante $h$, nos lleva $a$,

$$
\begin{gathered}
F(x+h, y) \simeq F(x, y)+\frac{\partial F(x, y)}{\partial x} h+\frac{\partial^{2} F(x, y)}{\partial x^{2}} \frac{h^{2}}{2}+\mathrm{O}\left(x^{3}\right) \\
F(x-h, y) \simeq F(x, y)-\frac{\partial F(x, y)}{\partial x} h+\frac{\partial^{2} F(x, y)}{\partial x^{2}} \frac{h^{2}}{2}-\mathrm{O}\left(x^{3}\right)
\end{gathered}
$$

Sumando estas dos ecuaciones miembro a miembro llegamos a la ecuación siguiente,

$F(x-h, y)-2 F(x, y)+F(x+h, y)=\frac{\partial^{2} F(x, y)}{\partial x^{2}} h^{2}+\left(h^{4}\right)$

En esta, el término $\left(h^{4}\right)$ es el error introducido por el truncamiento de ambas series. En general $\left(h^{4}\right)$ es muy pequeño y puede ser despreciado, resultado en la siguiente expresión que representa a las segundas derivadas parciales en términos de diferencias,

$$
\frac{\partial^{2} F(x, y)}{\partial x^{2}}=\frac{F(x-h, y)-2 F(x, y)+F(x+h, y)}{h^{2}}
$$

$$
\frac{\partial^{2} F(x, y)}{\partial y^{2}}=\frac{F(x, y-h)-2 F(x, y)+F(x, y+h)}{h^{2}}
$$

De la misma forma se desarrollan expresiones similares para la primeras derivadas parciales, resultando en

$$
\begin{aligned}
& \frac{\partial F(x, y)}{\partial x}=\frac{F(x+h, y)-F(x-h, y)}{2 h} \\
& \frac{\partial F(x, y)}{\partial y}=\frac{F(x, y+h)-F(x, y-h)}{2 h}
\end{aligned}
$$

Estas últimas son las ecuaciones que deben ser incluidas en la implementación del Método de Diferencias Finitas; las mismas han sido consideradas en el algoritmo que se detalla a continuación.

\section{B. Algoritmo}

El algoritmo en que se basa la aplicación del FDTD se describe en la secuencia siguiente:

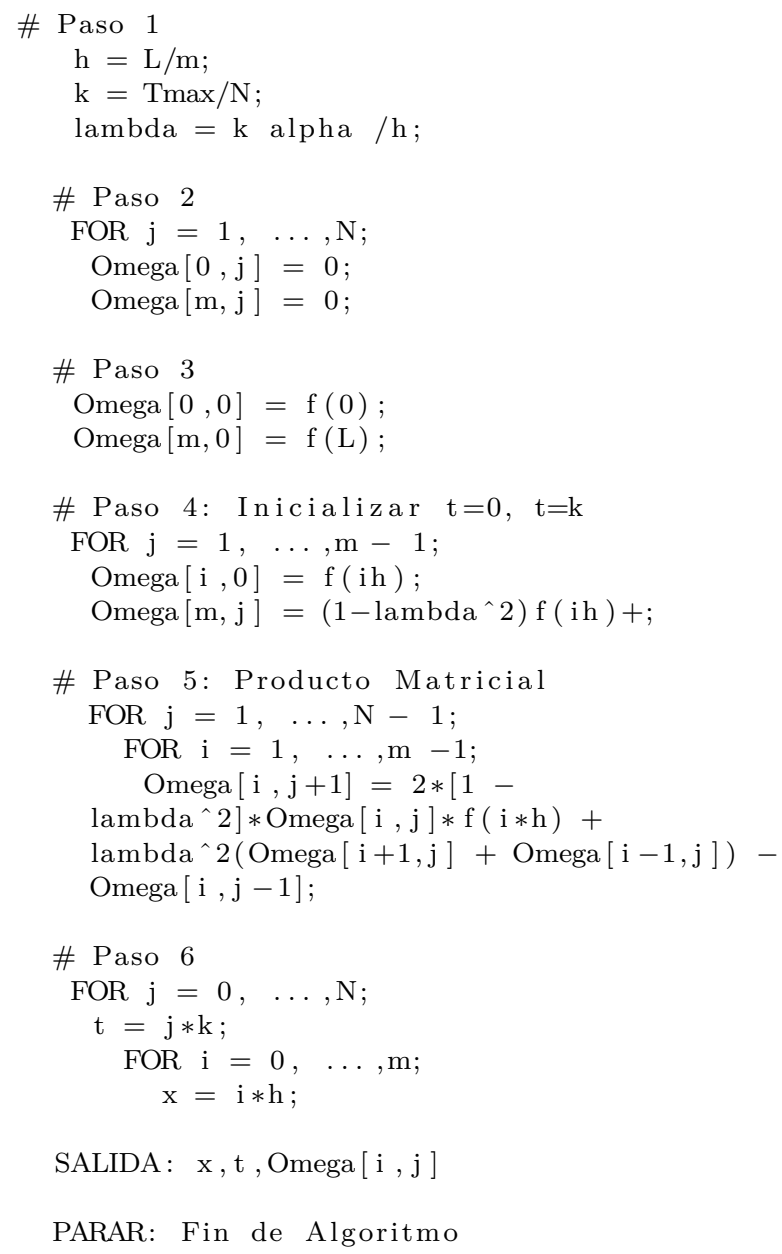

Debido a que EJS fue diseñado para proveer un ambiente de programación visual, el nivel de programación requerido para implementar este algoritmo es básico y la estructura es simple, pudiendo ser fácilmente implementado por estudiantes de pregrado en su primer curso de electromagnetismo.

\section{Solución Analítica}

Se plantea resolver la ecuación 8 , que corresponde a una ecuación diferencial en derivadas parciales del tipo hiperbólica. Esta ecuación está sometida a las siguientes 
condiciones iniciales y de frontera [15]:

- $U(0, t)=U(1, t)=0$, para $\mathrm{t} \geq 0$,

- $U(x, 0)=\operatorname{sen}(\pi / x), 0 \leq \mathrm{x} \leq 1$,

- $\delta U(x, 0) / \delta \mathrm{t}=0$, para $0 \leq \mathrm{x} \leq 1$

con los siguientes valores para los parámetros

- $h=0.1$ (incremento en $t)$,

- $k=0.05$ (incremento en $x$ ),

y con la siguiente solución analítica:

$$
U(x, t)=\operatorname{sen}(\pi x) * \cos (2 \pi t)
$$

D. Visualización Gráfica de Soluciones Numérica y Analítica

La figura 1 muestra la interfaz gráfica del usuario de FDTDM-EcOnda. En primera instancia despliega en la pantalla tanto la solución analítica como la solución numérica de la ecuación anteriormente planteada. La línea roja corresponde a la solución analítica y la azul a la solución numérica.

En segunda instancia, por debajo de la pantalla de visualización gráfica, se encuentra el panel de controles de la simulación, a través de estos, el usuario puede tener acceso a las tablas de datos generados por la simulación, y a la visualización gráfica bidimensional o tridimensional de las soluciones. IPS es un cursor con el que se selecciona el número de iteraciones por segundo que la computadora realiza. A mayor valor de IPS, menor velocidad de cálculo y despliegue de resultados obtenidos con el algoritmo.

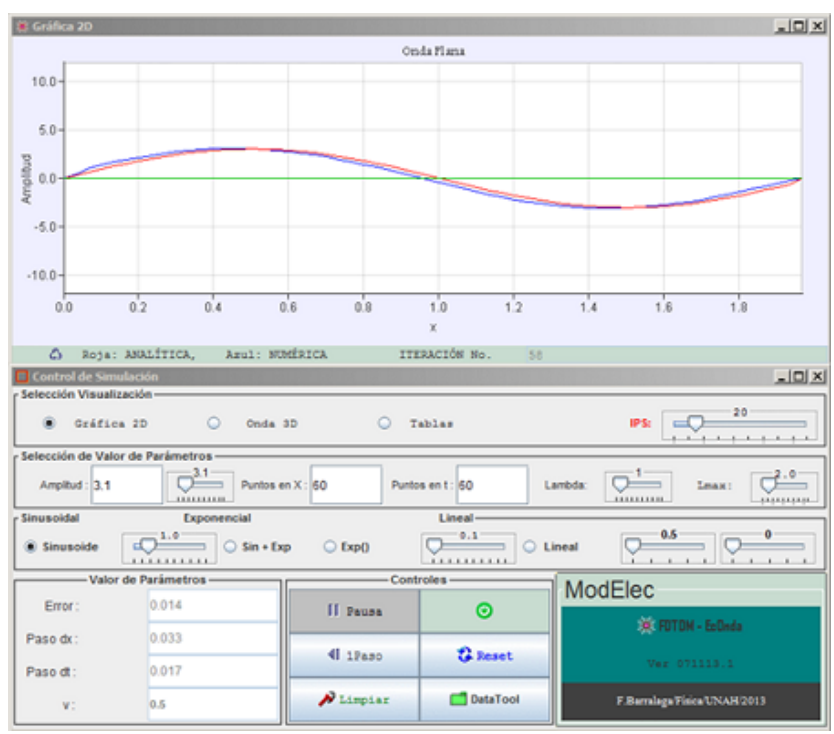

Figura 1: Interfaz de usuario. Visualización simultánea de ambas soluciones.

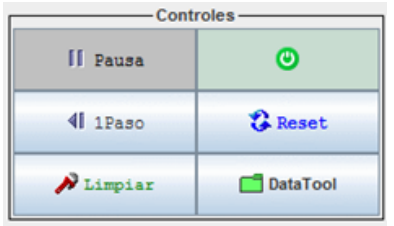

Figura 2: Controles.

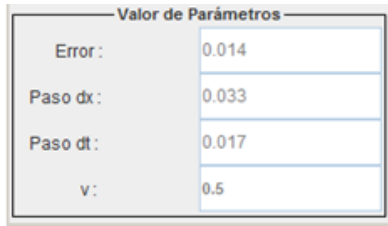

Figura 3: Parámetros.
La aplicación cuenta con opciones de control de ejecución con los que la simulación puede ser pausada, ejecutada en pasos unitarios o reinicializada a su estado de ejecución inicial. La figura 2 incluye esta ventana.

La ventana de valor de parámetros, muestra el cálculo aproximado de error, y los valores del paso de tiempo $d t$, y paso espacial $d x$. Un detalle de la ventana se muestra en la figura 3.

\section{E. Propiedades Gráficas y Control de Paráme- tros}

La visualización de bidimensional compara los resultados numéricos (línea azul) a lo largo de la longitud $L$ del eje $x$, en un instante $t$ cualquiera, con los correspondientes en ese mismo instante obtenidos con la solución analítica (línea roja). Obsérvese en la figura 4a a la solución numérica desplegada en un ambiente tridimensional susceptible a ser rotada por medio del cursor del ratón.

Si la amplitud de la onda es reducida, el efecto se visualiza inmediatamente como lo muestra la figura $4 \mathrm{~b}$ - La aplicación ofrece la posibilidad de cambiar los patrones que inicializan el algoritmo FDTM; así, es posible seleccionar opciones exponenciales, lineales, sinusoidales puros y la multiplicación de sinusoidal por exponencial. Por la simple comparación gráfica de ambas soluciones, resulta evidente que la mejor solución se consigue con la sinusoidal pura, cuya frecuencia puede ajustarse a la de la solución analítica.

El cambio de la frecuencia de la señal de perturbación inicial produce el efecto mostrado en la figura $5 \mathrm{a}$

\section{F. Datos Generados por Algoritmo}

La selección de la opción "Tablas" de la línea superior de la ventana de control ofrece al usuario los datos de la grilla espacio-temporal ( $x y t)$, y las amplitudes de la onda a lo largo del eje $x$.

Estos datos pueden ser exportados a una hoja de Excel con las opciones normales de copia y vertido de Windows.

\section{G. Módulo de Análisis Estadístico}

EJS provee un módulo de análisis estadístico y ajuste de curvas con el que los datos producidos por las aplicaciones pueden ser analizados y ajustados con modelos analíticos.

La figura 4c muestra detalle de la solución numérica calculada por el algoritmo de diferencias finitas de la 


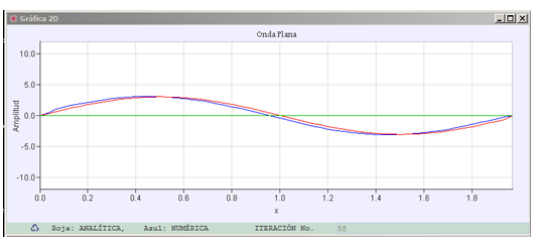

(a) Solución numérica y analítica.

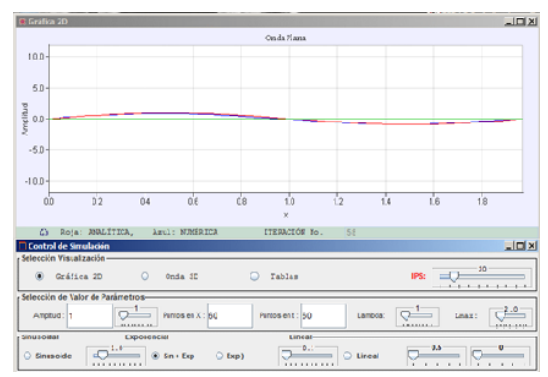

(b) Cambio de amplitud de la onda.

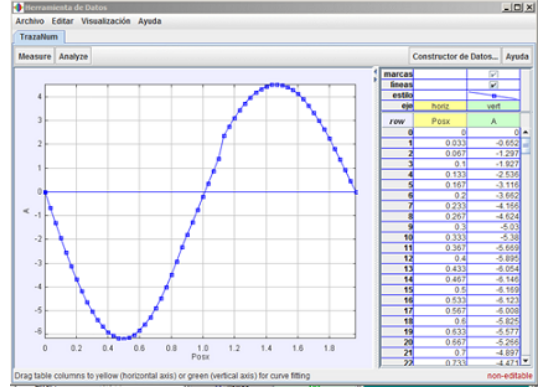

(c) Módulo de análisis estadístico.

Figura 4

aplicación en un instante $t$, y en la tabla de la derecha este módulo despliega los valores correspondientes. Se accede a este módulo pulsando el botón izquierdo del ratón con el cursor posicionado en la línea de datos cuyos valores se desea enviar al módulo de análisis. Para ello se requiere pausar antes la aplicación con la opción "Pause" de los controles de ejecución, o con la opción de "1Paso" de los mismos (ver figura 2).

La figura 6a incluye los valores analíticos de la onda en el mismo instante en que se generaron los valores numéricos correspondientes. Obsérvese que el módulo ha incluido una hoja (TrazaNum) con la información numérica generada por el algoritmo FDTDM, y otra hoja (TrazaMat) conteniendo los datos de la solución analítica.

Cuando se accede al sub-módulo Analyze, EJS realiza el cálculo del coeficiente de correlación entre los datos de numéricos y analíticos que han sido introducidos al módulo para su análisis. El detalle del resultado se muestra en la figura $6 \mathrm{~b}$. En parte inferior de la pantalla se observa el resultado del cálculo del coeficiente de correlación de este ejemplo, $(R-$ squared $=0.977)$, lo que demuestra que la solución numérica producida por el método de diferencias finitas con dominio del tiempo, genera una muy buena aproximación a la solución analítica.

\section{CONCLUSIONES}

- El nivel de correlación obtenido implica que el Método de Diferencias Finitas con Dominio del Tiempo, implementado con el algoritmo mencionado, genera una solución numérica de la ecuación de onda unidimensional con resultados muy satisfactorios.

- EJS es una opción suficientemente poderosa para obtener soluciones numéricas de la ecuación de onda unidimensional y generar el despliegue gráfico de esta en tiempo real, con un nivel de programación que no requiere gran esfuerzo ni excesivo tiempo de programación. EJS es una herramienta didáctica adecuada en procesos de simulación por computadora, de fenómenos relacionados con el electromagnetismo.

- La aplicación FDTDM-EcOnda muestra los efectos resultantes de variar el valor de los parámetros del algoritmo. Así es evidente que el uso de patrones iniciales diferentes a una señal sinusoidal aleja la solución numérica de la analítica. Con estas propiedades de visualización simultánea de ambas soluciones, la aplicación resulta de gran ayuda didáctica complementaria en el estudio de la solución de onda unidimensional.

\section{REFERENCIAS}

[1] Antonio Nieves, F. C. (1995). Métodos numéricos aplicados a la ingeniería. México: CECSA.

[2] Beatriz Elena Osorio Vélez, L. S. M. A. J. A. O. V. G. E. C. F. R. C. (2012). Análisis de la enseñanza y el aprendizaje del eelectromagnetismo en el nivel tecnológico y universitario. Entre Ciencia y Ingeniería, $1(12)$.

[3] Bloom, B. S. (1984). The 2 sigma problem: The search for methods of group instruction as effective as one-to-one tutoring. Educational Researcher, 13(6), 416. URL http://www. jstor.org/stable/1175554.

[4] Burd, B. (2005). Beginning Programming with JAVA. Wiley Publishing, Inc.

[5] Dormido, S.; Farias, G.; Sanchez, J. y Esquembre, F. (2005). Adding interactivity to existing simulink models using easy java simulations. En Proceedings of the 44th IEEE Conference on Decision and Control, (4163-4168), doi:10.1109/CDC.2005.1582815.

[6] Esquembre, F. (2009), Manual de ejs. Universidad de Murcia.

[7] Esquembre, F. (2004). Easy java simulations: a software tool to create scientific simulations in java. Computer Physics Communications, 156(2), 199 204, doi:http://dx.doi.org/10.1016/S0010-4655(03) 00440-5. URL http://www.sciencedirect.com/ science/article/pii/S0010465503004405.

[8] Fawwaz, U. (2002). Fundamentos de aplicaciones en electromagnetismo (5ta. (Español) ed.). Pearson, Prentice Hall. 

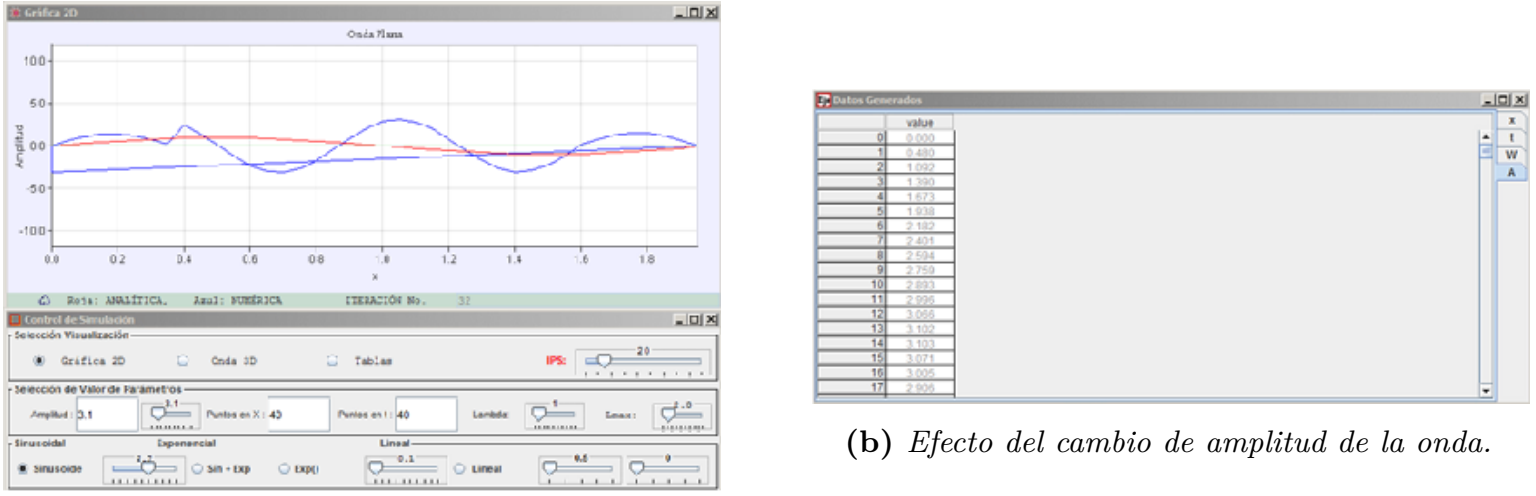

(b) Efecto del cambio de amplitud de la onda.

(a) Cambio de frecuencia en perturbación.

Figura 5

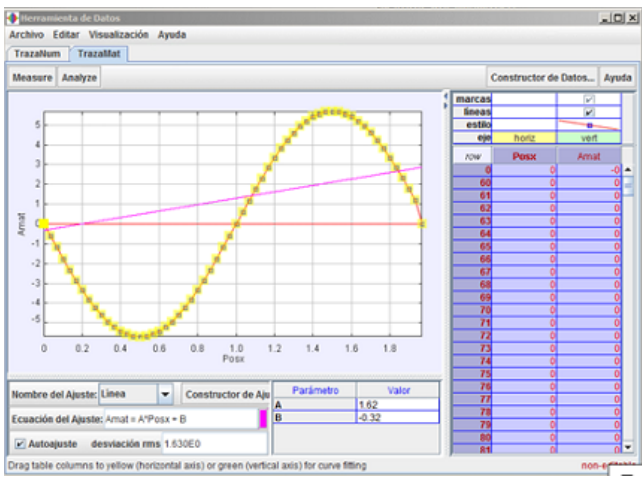

(a) Datos analíticos en un instante t.

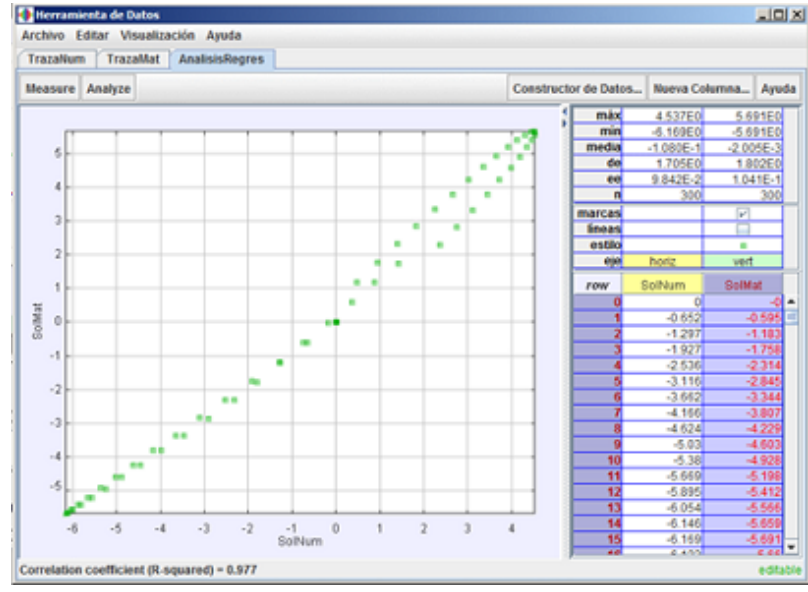

(b) Correlación entre datos analíticos y numéricos producidos con la aplicación del Método de Diferencias Finitas con Dominio del Tiempo, FDTD.

Figura 6

[9] Gould, H.; Tobochnik, J. y Wolfgang, C. (2005). An Introduction to Computer Simulation Methods: Applications to Physical Systems (3rd Edition). Boston, MA, USA: Addison-Wesley Longman Publishing Co., Inc.

[10] Griffiths, D. J. (1999). Introduction to Electrodynamics. McGraw Hill.

[11] Guisasola, J.; Almudí, J. M. y Zubimendi, J. L. (2004). Difficulties in learning the introductory magnetic field theory in the first years of university. Science Education, 88(3), 443-464, doi:10.1002/sce.10119. URL http://dx.doi .org/10.1002/sce.10119.

[12] Herrera, L. J. (2010), Apuntes del curso oscilaciones y ondas. Recuperado de: http://www.fisica.ru/dfmg/teacher/archivos/ movimientooscilatorio.pdf

[13] Marti, A. U. (2007-8), Aplicación de la simulación por ordenador a la enseñanza de las ciencias (uned). Recuperado de: http://www.euclides.dia.uned.es/ aurquia/Files/textoBaseEjs.pdf.

[14] Nagornov, C. R. (2001). Animando la física partei: Propagación de ondas. Revista Argentina de
Enseñanza de la Ingeniería, 1(3), 39-44. URL http://www.ing.unrc.edu.ar/raei/archivos/ img/arc_2011-11-27_16_50_18-35.pdf.

[15] Richard L. Burden, J. D. (1985). Análisis Numérico. Grupo Editorial Iberoamericano.

[16] Sadiku, M. (2000). Elements of Electromagnetism. Oxford University Press.

[17] Urdaneta, M. (2007). Aplicación del método de diferencias finitas en el dominio del tiempo para la simulación de la propagación de ondas electromagnéticas en ambientes cerrados. Tesis, Universidad de Zulia, Maracaibo.

[18] Wangsness, R. (2001). Campos Electromagnéticos. Limusa.

[19] Zozaya, S. A. (2004), Método de las diferencias finitas y su aplicación a problemas de electrostática. Recuperado de: http://www.ing.uc.edu.ve/ azozaya/ docs/tem1/DF.pdf 